\title{
Electropolymerized Molecular Imprinting \& Graphene Modified Electrode for Detection of Melamine
}

\author{
Zhe $\mathrm{Ji}^{1,2}$, Wen Chen $^{1,2, *}$, Er Wang, ${ }^{1,2}$, and Rongriu Deng ${ }^{1,2}$ \\ ${ }^{1}$ College of Materials and Chemistry \& Chemical Engineering, Chengdu University of Technology, \\ Chengdu, Sichuan 610059, People's Republic of China \\ ${ }^{2}$ Mineral Resources Chemistry Key Laboratory of Sichuan Higher Education Institution, Chengdu, \\ Sichuan 610059, People's Republic of China \\ *E-mail: chenwen2010@foxmail.com
}

doi: $10.20964 / 2017.12 .34$

Received: 30 July 2017 / Accepted: 19 October 2017 / Published: 12 November 2017

\begin{abstract}
A new method for the determination of melamine (MEL) indirectly by the changes of differential pulse voltammetry (DPV) current of $\mathrm{K}_{3}\left[\mathrm{Fe}(\mathrm{CN})_{6}\right]$ probes after 10 min incubation $(\Delta \mathrm{I})$ has been established. It's based on the inhibitory effect of MEL on occupying the molecular recognition sites of surface of the electropolymerized molecular imprinting \& graphene modified glassy carbon electrode (MIPPy/GR/GCE). The MIPPy/GR/GCE was characterized by cyclic voltammetry (CV), electrochemical impedance spectrometry (EIS) and scanning electron microscopy (SEM). The $\Delta \mathrm{I}$ is linearly proportional to the negative logarithmic concentration of MEL over the range of $3.0 \times 10_{-8}$ $\sim 1.0 \times 10_{-4} \mathrm{~mol} \cdot \mathrm{L}^{-1}(\mathrm{R}=0.9948)$, the detection limit is $1.02 \times 10^{-8} \mathrm{~mol} \cdot \mathrm{L}^{-1}(\mathrm{~S} / \mathrm{N}=3)$. The recoveries of samples are from $102.65 \%$ to $108.02 \%$, the result is satisfactory when it is used to analyze the real samples.
\end{abstract}

Keywords: Molecularly imprinted polymer; Graphene; Melamine; Sensor

\section{FULL TEXT}

(C) 2017 The Authors. Published by ESG (www.electrochemsci.org). This article is an open access article distributed under the terms and conditions of the Creative Commons Attribution license (http://creativecommons.org/licenses/by/4.0/). 